\title{
Characterizing the developmental transcriptome of the oriental fruit fly, Bactrocera dorsalis (Diptera: Tephritidae) through comparative genomic analysis with Drosophila melanogaster utilizing modENCODE datasets
}

Scott M Geib ${ }^{1 *}$, Bernarda Calla ${ }^{1}$, Brian Hall ${ }^{1,2}$, Shaobin $\mathrm{Hou}^{3}$ and Nicholas C Manoukis ${ }^{1}$

\begin{abstract}
Background: The oriental fruit fly, Bactrocera dorsalis, is an important pest of fruit and vegetable crops throughout Asia, and is considered a high risk pest for establishment in the mainland United States. It is a member of the family Tephritidae, which are the most agriculturally important family of flies, and can be considered an out-group to well-studied members of the family Drosophilidae. Despite their importance as pests and their relatedness to Drosophila, little information is present on B. dorsalis transcripts and proteins. The objective of this paper is to comprehensively characterize the transcripts present throughout the life history of $B$. dorsalis and functionally annotate and analyse these transcripts relative to the presence, expression, and function of orthologous sequences present in Drosophila melanogaster.

Results: We present a detailed transcriptome assembly of $B$. dorsalis from egg through adult stages containing 20,666 transcripts across 10,799 unigene components. Utilizing data available through Flybase and the modENCODE project, we compared expression patterns of these transcripts to putative orthologs in D. melanogaster in terms of timing, abundance, and function. In addition, temporal expression patterns in B. dorsalis were characterized between stages, to establish the constitutive or stage-specific expression patterns of particular transcripts. A fully annotated transcriptome assembly is made available through NCBI, in addition to corresponding expression data.

Conclusions: Through characterizing the transcriptome of $B$. dorsalis through its life history and comparing the transcriptome of $B$. dorsalis to the model organism D. melanogaster, a database has been developed that can be used as the foundation to functional genomic research in Bactrocera flies and help identify orthologous genes between B. dorsalis and D. melanogaster. This data provides the foundation for future functional genomic research that will focus on improving our understanding of the physiology and biology of this species at the molecular level. This knowledge can also be applied towards developing improved methods for control, survey, and eradication of this important pest.
\end{abstract}

\footnotetext{
*Correspondence: scott.geib@ars.usda.gov

'Tropical Crop and Commodity Protection Research Unit, USDA-ARS Daniel K Inouye Pacific Basin Agricultural Research Center, 64 Nowelo Street, Hilo, HI 96720, USA

Full list of author information is available at the end of the article
} 


\section{Background}

The oriental fruit fly (Bactrocera dorsalis) is an important agricultural pest in Asia, Africa, and the Pacific, impacting over 150 fruits and vegetables in tropic and sub-tropic regions $[1,2]$. In addition, $B$. dorsalis is established across the Hawaiian Islands and is a species of concern in the continental United States, with numerous interceptions and detections made annually, often triggering eradication efforts and quarantines [3-5]. In addition to being an important pest species, $B$. dorsalis, as a member of the family Tephritidae, can serve as an important species for comparison to the well characterized drosophilid group, having diverged from Drosophila approximately 70 million years ago [6].

Despite its importance as an agricultural pest, little functional genomic information is available for the species outside of a collection of sequences used for phylogenetic analysis of the genus. The addition of functional genomics information can lead to development of new control strategies, a basic understanding of the biology of the insect, and expansion of the techniques used in research. With the advent of high-throughput sequencing technology, there is increased ability to obtain cost-effective sequence data from non-model organisms, including sequencing from total RNA libraries to target coding genes at particular time points. While collection of this data is fairly straightforward, in order to accurately predict gene models and transcripts, much attention must be given towards assembly and analysis of the data. While this shotgun de novo approach to sequencing potentially allows for collection of full length genes, detection of splice variants, and calculation of differential expression between tissues, it can also lead towards assembly of partial gene fragments, erroneous assembly fragments, and mis-assemblies [7-9]. Many RNA-seq experiments report a large number of small transcript fragments, sometimes numbering in the hundreds of thousands, with a small proportion likely to be full length transcripts. This can make it difficult to calculate meaningful expression values, accurately identify transcript isoforms, and limit the utilization of the resulting dataset in downstream functional genomics experiments. For example, for $B$. dorsalis there are several published experiments that have performed de novo transcriptome assembly, presented tens of thousands of assembled contigs, but did not publish gene models or transcript sequences as part of their experiment [10-12]. This makes it difficult to utilize the research and limits the application of the research. As an alternative, we attempted to analyze RNA-seq data to produce a high confidence transcript set consisting of full length or near full-length transcripts with strong support for their accuracy. The resulting dataset can be used as a foundation for expanding on the $B$. dorsalis gene set without concerns that the dataset contains erroneous data.
Our approach was to perform comprehensive RNA sequencing on a laboratory colony of $B$. dorsalis with a focus on attempting to capture expression of as many genes and gene splice variants that represent the entire developmental life history. De novo transcriptome assembly and analysis of the resulting sequence were performed with a focus on identifying full length gene and splice variants, and then annotating through identification of orthologs in Drosophila gene sets. High confidence genes were filtered from potentially erroneously assembled transcripts based on homology to known proteins and read coverage of the transcript. The result produced a high quality reference transcript set for this species for comparative analysis with $D$. melanogaster. This transcriptome can be used as a foundation for functional genomic and population genetic experiments. Further development from other lines of evidence can be used in the future to broaden this gene set.

\section{Results and discussion}

\section{Sequencing and quality filtering}

In total, approximately 101 million paired $100 \mathrm{bp}$ reads were obtained from Illumina GAIIx sequencing, totalling over $20 \mathrm{~Gb}$ of data (Table 1). These reads were evenly distributed between all of the libraries sequenced (egg, larvae, pupae, adult male, adult female, mated adult female). All raw reads were submitted to the NCBI Sequence Read Archive under accession numbers SRX261507-SRX261512 associated with BioProject PRJNA167923. After quality filtering and removing low quality reads, approximately $84 \%$ of reads remained and were used for assembly and mapping. While filtering provided little improvement to the quality of the beginning of the read, it dramatically increased the quality score of the end of the read from an average q20 score of 24.9 at base 95 in unfiltered reads to average q20 score of 29.2 in filtered reads (Table 1). Filtering also had no dramatic effect on GC content of the data, suggesting there was no bias to the reads that were removed during filtering. In silico normalization using the Trinity normalization tool dramatically reduced the number of input reads into the assembly without adversely influencing the kmer abundance from those reads. From $\sim 101,364,649$ raw read pairs, filtering and normalization reduced the read abundance to 7,796,491 ( 7.7\%) reads used as input into the Trinity assembly, greatly reducing the computational requirements for assembly and avoiding complicated de bruin graphs created by low quality sequence or overabundant sequence kmers.

\section{De novo transcriptome assembly and transcript filtering}

The $B$. dorsalis transcriptome was reconstructed using the Trinity assembly pipeline with all filtered reads from all libraries pooled into one dataset $[9,13]$. This raw 
Table 1 Quality filtering of Illumina GAllx data before assembly and mapping

\begin{tabular}{|c|c|c|c|c|c|c|c|c|c|c|c|c|c|c|c|c|c|c|c|c|c|}
\hline & & & & \multicolumn{12}{|c|}{ Average quality (interquartile range) } & \multicolumn{6}{|c|}{ GC content (percent) } \\
\hline & \multicolumn{3}{|c|}{ Number of reads } & \multicolumn{6}{|c|}{ Before filtering } & \multicolumn{6}{|c|}{ After filtering } & \multicolumn{3}{|c|}{ Before filtering } & \multicolumn{3}{|c|}{ After filtering } \\
\hline & Before filtering & After filtering & Percent retained & Bas & e 5 & Base & e 50 & Base & 95 & Base & & Bas & e 50 & Bas & e 95 & Base 5 & Base 50 & Base 95 & Base 5 & Base 50 & Base 95 \\
\hline Egg & 29899982 & 24924408 & $83.4 \%$ & 36.7 & (4) & 34.1 & (7) & 24.9 & (25) & 37.7 & (2) & 36.6 & (5) & 29.3 & (8) & 40.0 & 41.1 & 41.9 & 38.9 & 39.8 & 40.6 \\
\hline Larvae & 28913860 & 23506167 & $81.3 \%$ & 36.7 & (4) & 33.8 & (6) & 23.5 & (33) & 37.7 & (2) & 36.4 & (5) & 28.2 & (8) & 41.2 & 42.8 & 44.2 & 40.1 & 41.4 & 42.8 \\
\hline Pupae & 31954342 & 26582294 & $83.2 \%$ & 36.8 & (4) & 34.2 & (7) & 24.6 & (33) & 37.7 & (2) & 36.6 & (5) & 29.0 & (8) & 40.6 & 42.0 & 42.8 & 39.4 & 40.6 & 41.4 \\
\hline Male & 41546048 & 35350086 & $85.1 \%$ & 37.4 & (2) & 35.1 & (5) & 25.8 & (17) & 38.2 & (2) & 37.3 & (4) & 29.8 & (7) & 39.7 & 41.5 & 42.4 & 38.5 & 40.0 & 41.1 \\
\hline Female & 35096592 & 29240854 & $83.3 \%$ & 36.9 & (4) & 34.4 & (7) & 24.7 & (33) & 37.8 & (2) & 36.7 & (5) & 29.0 & (8) & 39.7 & 41.9 & 42.8 & 38.5 & 40.5 & 41.4 \\
\hline Mated Female & 35318474 & 29909305 & $84.7 \%$ & 36.9 & (4) & 34.5 & (7) & 25.3 & (18) & 37.8 & (2) & 36.7 & (5) & 29.4 & (8) & 38.5 & 40.9 & 41.9 & 37.4 & 39.6 & 40.8 \\
\hline Total & 202729298 & 169513114 & $83.6 \%$ & 36.9 & (3.5) & 34.4 & $(6.5)$ & 24.9 & (26) & 37.8 & (2) & 36.7 & (4.8) & 29.2 & $(7.8)$ & 39.9 & 41.7 & 42.6 & 38.7 & 40.3 & 41.3 \\
\hline
\end{tabular}


assembly yielded 80,346 contigs, with an N50 contig size of 2,802 bases, 31,321 contigs greater than $1000 \mathrm{bp}$, and a transcript sum of 109.4 Mb. While not all assembled contigs produced by Trinity represent true transcripts in $B$. dorsalis, this contig set was used as a starting point for defining the transcriptome present in our sample. Filtering based off of read abundance and component isoform percentage removed 35,729 sequences, leaving 44,617 remaining. Further filtering through identification of likely coding sequence based on ORF prediction identified 6,864 genes containing a complete ORF (unique genes defined as sequences with unique comp\#\#_c\# identifier from Trinity (http://trinityrnaseq.sourceforge.net/)) with 13,017 isoforms giving complete ORFs. In addition, 3,935 genes only contained a partial ORF (missing either 5 ' or $3^{\prime}$ end or both) with 7,649 isoforms identified in those genes, giving a total of 20,666 transcripts across 10,799 genes, with an N50 transcript size of 3,460 bp and transcript sum of 62.08 Mb. While Trinity assembled an additional 56,785 contigs, these were discarded by the above filtering because they lacked a likely coding sequence or did not have significant read coverage. While in some cases these may contain partial gene fragments, we chose to exclude them from further analysis as the majority consist of short, low read count data with low blast homology to known proteins in D. melanogaster (Figure 1A-C). In contrast, the majority of the retained transcripts had a significant number of reads mapping to them, with full length transcripts being longer, and having a higher alignment percentage to known Drosophila proteins when compared to the partial transcripts (Figure 1A-C).

\section{Read abundance based expression analysis across developmental stages}

Stage specific expression values were calculated for each developmental library as TMM normalized FPKM values. Unique genes were classified with the modENCODE FPKM expression categories (very low, low, moderate, moderate high, high, very high, extremely high) utilized by flybase.org with the same FPKM expression level calculations and category bins used in Drosophila [14,15]. Distribution of expression categories across developmental stages are presented in Figure 2. From this, genes were categorized as constitutively expressed in all libraries, stage-specific or expressed across several stages. Constitutive highly expressed genes were defined as those genes having an expression level of "moderately high" or higher in all 6 samples. Under this definition, we identified 1,347 constitutive highly expressed genes in our dataset. Stage specific genes were defined as those that are "moderately expressed" or higher in one library and "low" or "very low" in the other 5 libraries. A total of 310 unigenes were declared stage specific. Finally, genes expressed across two, three, four, or five libraries were also calculated, as many genes may be expressed during several related libraries that could constitute a biologically relevant developmental stage. This identified 395 genes expressed at least moderately high in two libraries compared to very low in the rest of the libraries, 186 genes with this same pattern in three libraries, 156 in 4 libraries and 136 in 5 libraries. The majority of the genes in the combined transcriptome dataset were found constitutively expressed at a low level ("low" or "very low", 8,269 genes). A breakdown of the number of stage specific genes is listed in Table 2, and a matrix of stage specific and constitutively expressed genes is provided in Additional file 1.

Using the same classification described above, the number of genes falling into each of the expression level categories for each stage was compared with that of D. melanogaster (Figure 3), demonstrating strong similarity of distribution of gene expression levels in both species. While this result helps to corroborate the expression values presented for $B$. dorsalis, it additionally highlights the intra-species conservancy of expression levels for the majority of transcripts, suggesting stage-specific functional conservation between the identified genes in $B$. dorsalis and D. melanogaster (Figure 3).

Another complementary approach to compare expression between stages was employed through hierarchical clustering of the gene expression values between libraries, and then defining subclusters that share similar expression patterns across libraries. From the hierarchical clusters, 19 subclusters were created by splitting the tree at clusters that shared $45 \%$ of the tree height. These subclusters were visualized by plotting the median centered FPKM values (Figure 4). In this case, expression patterns are not focused specifically on the most highly expressed genes as in the first approach, but rather on genes that exhibit a change in expression based on median-centered values, regardless of the level of expression. Some of the defined clusters did not clearly describe a stage specific expression pattern such that no expression values were distributed significantly outside of the median (e.g. cluster Q, Figure 4); others, however, showed clear differences in expression between libraries. These patterns are highlighted as inset figures in Figure 4, and a list of genes present in each cluster is provided as supplementary data (Additional file 2).

\section{Putative unigenes orthologous to $D$. melanogaster genes}

A set of 5,681 putative orthologous genes were identified in our $B$. dorsalis dataset through reciprocal BLAST alignment with the $D$. melanogaster protein set. We anticipate this number to be an underestimation of actual ortholog representation, as utilizing an RNA-seq assembly increases the chance for multiple unigenes. Expression levels in terms of normalized FPKM values were compared between the putative orthologs by plotting the data along a regression 

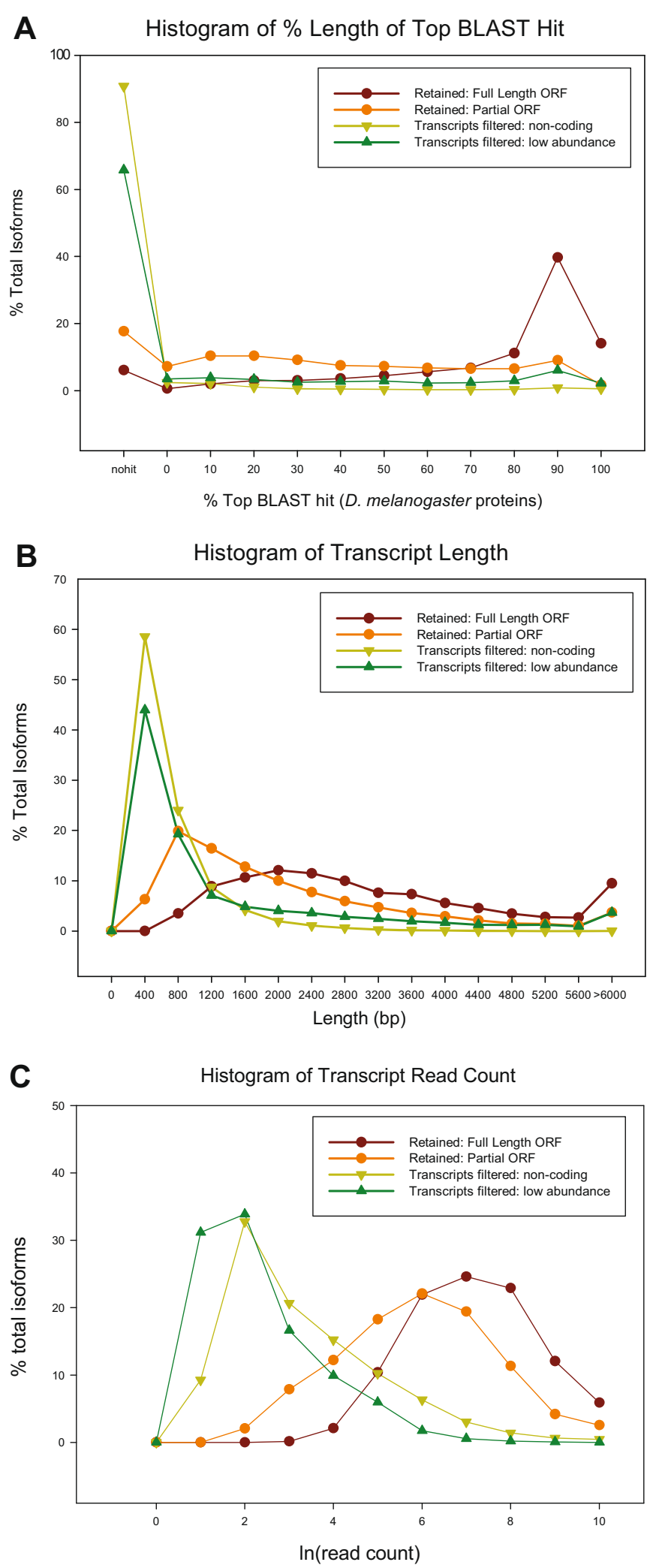

Figure 1 (See legend on next page.) 
(See figure on previous page.)

Figure 1 Comparative evidence for filtering of de novo assembly. Lines represent retained unigenes (either containing a full or partial ORF) or transcripts discarded based off of lack of evidence of coding region or due to low expression values. A) Proportion length of $B$. dorsalis putative transcripts relative to highest scoring blast alignment to D. melanogaster proteins. B) Length distribution of transcripts. C) Relative log read count expression values of transcripts.

curve. A strong linear relationship was observed between the expression values of orthologs (Figure 5). To identify orthologous proteins with potential difference in expression between the two species, a 95\% interval was calculated along the regression. A conservative approach was taken to avoid the identification of false orthologs, and at times this approach may not identify true orthologs. This is particularly true when using de novo assembled RNA-seq data, which commonly has a large proportion of extraneous or superfluous transcripts assembled due to genetic heterogeneity of the sample, sequence error, and/ or relaxed transcript reconstruction parameters of the software. By filtering the assembly to the approximate expected unigene and transcript density, over 5,600 putative orthologs were identified. Through linear regression analysis between expression values of the orthologs in $B$. dorsalis and D. melanogaster, we found a strong linear correlation at each developmental stage. Of the unigenes falling outside of the confidence interval of the regression, a significant proportion were identified as constitutive low expression. This bias seems largely due to a large number of unigenes with higher expression (although still constitutively low) in $B$. dorsalis compared to $D$. melanogaster and are likely not biologically relevant. In contrast, there are 4,234 unigenes $(\sim 75 \%)$ that are always within the confidence interval and about 25\% that are conversely outside in at least one developmental stage (Additional file 3). Only 30 unigenes were consistently outside of the confidence interval in all 5 developmental stages. This strong homology in expression patterns between $B$. dorsalis and $D$. melanogaster for the putative orthologs suggests conserved functions between species. Of course, this is biased, as additional unigenes that may be orthologs were not identified by our analysis, and it is possible that those may be enriched for proteins that have modified function or expression patterns, and may not have passed the threshold set. Despite some genes not falling within the confidence interval of the regression, Pearson correlation values (rho) at a $\mathrm{p}<0.001$ were calculated and both data sets were found to have a strong correlation. Both the regression and the correlation support assembly accuracy and relative completeness. For genes outside of the confidence, while considered potential orthologs, may have different functions or temporal expression patterns

\section{Stage Specific modEncode Expression Categories}

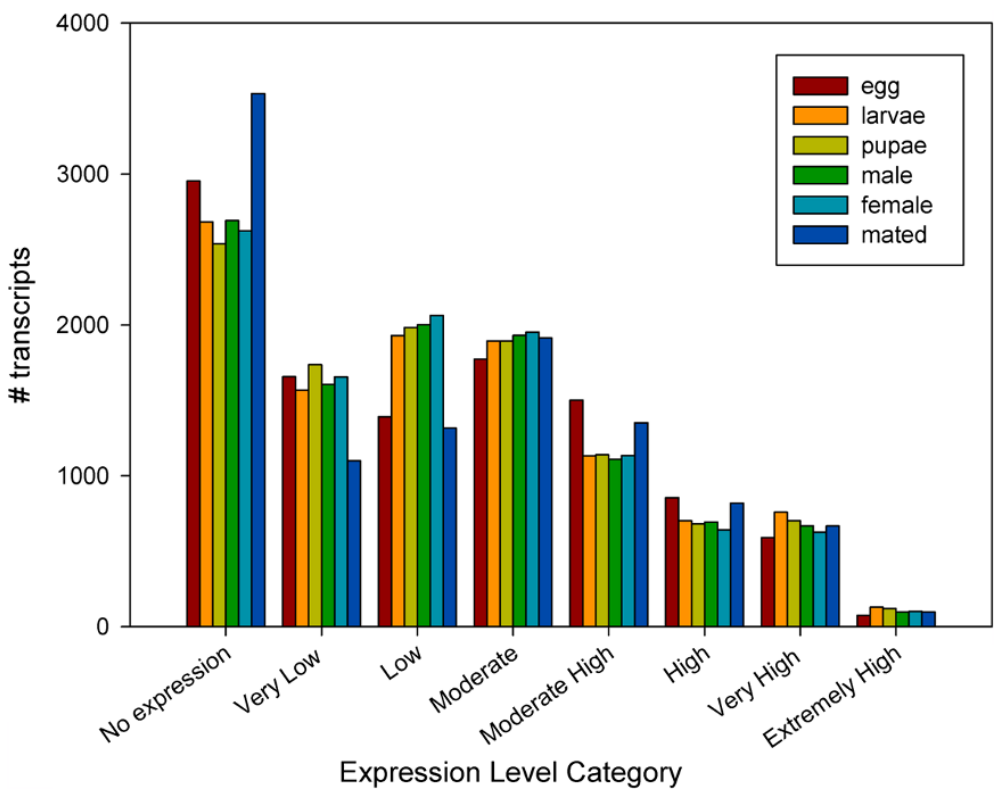

Figure 2 Distribution of expression categories across $\boldsymbol{B}$. dorsalis developmental stages. Placement of unigenes across modENCODE expression categories, separated by developmental stage. 
Table 2 Number of unigenes present in expression level categories

\begin{tabular}{lcc}
\hline Stage & $\begin{array}{c}\text { Number of } \\
\text { unigenes }\end{array}$ & $\begin{array}{c}\text { Percentage of total } \\
\text { unigenes (\%) }\end{array}$ \\
\hline All stages: & & \\
Constitutive highly expressed genes & 1,347 & 12.47 \\
Constitutive low & 8,269 & 76.56 \\
Single Stages: & & \\
Egg specific & 29 & 0.26 \\
Larvae specific & 136 & 1.25 \\
Pupae specific & 110 & 1.01 \\
Mated Female specific & 35 & 0.324 \\
Adults (Female and male) specific & 50 & 0.46
\end{tabular}

Lists of genes present in each category is provided as matrices in Additional file 1. Constitutive highly expressed genes have modENCODE expression values of "High" or greater across all stage specific samples. Sparsely expressed genes have a value of "Low" or lower.

between the two species, or may be more sensitive to external variables that potentially differed between rearing conditions.

\section{Functional annotation}

A large proportion of the transcripts assembled and retained after filtering were able to be functionally annotated through BLAST homology to proteins in the UniProt Swiss-Prot database and the D. melanogaster protein set or HMM profiles in Pfam-A. Further, when possible, gene ontology, KOG, and COG terms were added. In total, 17,093 transcripts (72.5\% of total) were annotated from BLAST homology to Swiss-Prot with a qualified gene name, whereas 20,713 transcripts ( $87.9 \%$ of total) had significant alignment to $D$. melanogaster proteins (r5.44), encompassing 10,686 proteins from 9,004 unique gene models in D. melanogaster, and representing $65 \%$ of known $D$. melanogaster genes. In contrast, 16,612 transcripts were annotated with Pfam domains, encompassing 3,846 unique Pfam identities.

\section{Identification of transcription factors in the $B$ dorsalis genome}

Spatial and temporal patterns of gene expression are in part regulated by transcription factors. Transcription factors (TFs) were searched in our dataset initially by using the Gene Ontology annotations and further curated using Pfam. Genes with GO terms corresponding to: GO:0003700 (sequence-specific DNA binding transcription factor activity), GO:0006355 (regulation of transcription, DNA-templated), and GO:0003705 (RNA polymerase II distal enhancer, sequence-specific DNA binding transcription factor activity) were selected and Pfam annotations were used to curate and classify the selected list of genes. A total of 1,265 transcripts were classified as TFs, roughly $6 \%$ of the transcriptome. This percentage is comparable with the number of TFs estimated in D. melanogaster [16]. The 1,265 identified sequences corresponded to one of 305 unique domains. The most abundant TFs corresponded to Zinc finger domains, followed by homeobox domains and helix-loop-helix proteins (Table 3). It is worth noting that several sequences may be false positives. For the purposes presented here, transcription factors were defined as DNA binding domains, however several proteins exist that possess motifs similar to DNA binding factors and yet may be unrelated. Similarly, several true transcription factors might be missing from the analysis.

\section{Detoxification genes in $B$. dorsalis transcriptome}

An important aspect of insect evolutionary adaptation is their ability to remove acquired toxic compounds and protect the organism. To identify genes related to detoxification in $B$. dorsalis, genes linked with Phases I and II in mammalian detoxification pathways were mined from the transcriptome dataset through sequence homology. Cytochrome P450 monooxygenases (P450s) accounted for 163 transcripts in 93 unique unigenes, approximately equating the 90 genes found in Drosophila from this superfamily [17]. These 93 unigenes belong to 12 families (Table 4). While many P450 proteins are known to be involved in detoxification, the superfamily consists of a wide array of enzymes with distinct functions $[17,18]$. This is not the first report of characterization of P450's in $B$. dorsalis, with previous research reporting as many as 90 unigenes to as few as 51 from shotgun transcriptome experiments $[10,12,19]$. Inconsistent database deposition of sequences obtained in previous reports makes it difficult to make comparisons and draw conclusions across the respective studies. In one case, multiple P450s were verified through cloning [20], and 60 P450's were reported from the olive fly $B$. oleae [21]. The overall consensus suggests strong consistency in the diversity and abundance of P450's in B. dorsalis and D. melanogaster.

\section{Glutathione-S-transferases (GSTs) and UDP-glucuronosyltransferases}

(UGTs) constitute phase II of the mammal detoxification pathway, usually by mitigating the effects of the oxygen radicals produced by phase I [22]. Our transcriptome profiling detected 34 unique GSTs domains, 26 of which had both $\mathrm{N}$ - and $\mathrm{C}$ - terminal domains and eight with homology to either the $\mathrm{C}$-terminal region only or the $\mathrm{N}$-terminal region (four and four respectively). This is comparable to a previous report that identified 37 unigenes with GST domains [12]. Additionally, 23 UGTs were found (Table 4). Furthermore, data from a D. melanogaster microarray experiment designed to profile genes in the CncC/Keap1 pathway was obtained [23]. The CnC/Keap pathway is presumably the central pathway for detoxification 


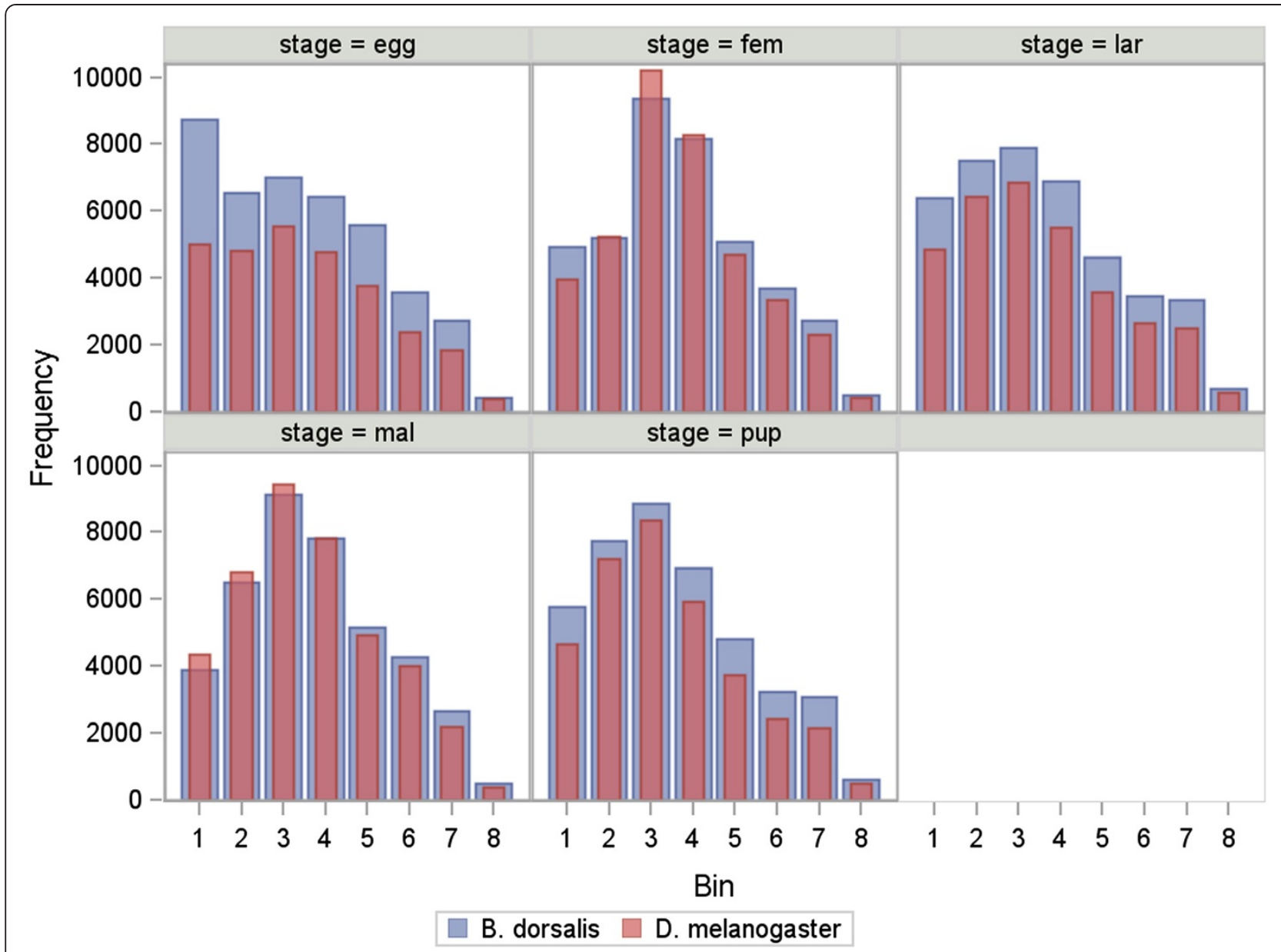

Figure 3 Comparison of expression level distribution between $B$. dorsalis and $D$. melanogaster utilizing modENCODE expression categories. Numbers below bars represent modENCODE expression categories as follows: 1- No Expression, 2- Very Low, 3- Low, 4- Moderate, 5- Moderate High, 6- High, 7-Very High, 8- Extremely High. Frequency of all B. dorsalis unigene models were compared to all gene level expression values derived from the $D$. melanogaster modENCODE project.

in D. melanogaster and has little evolutionary relationship with detoxification in mammals. The data was cross-referenced with our identified orthologous genes in $B$. dorsalis. Among the genes that changed in expression in response to $\mathrm{CncC} /$ Keap pathway activation in D. melanogaster, 141 appear to be present in B. dorsalis with most constitutively expressed (either at a high or low degree) across all stages, with no specificity to their expression pattern (Additional file 4).

\section{Sex determination transcripts}

Genes involved in sex-determination can be used to develop pest control tools as well as to improve the existing sterile insect technique (SIT) [24]. To identify sex-determinant genes, amino acid sequences of 67 transcripts corresponding to 14 genes known to be involved in sex determination in D. melanogaster $[25,26]$ were used to search our $B$. dorsalis transcriptome dataset trough BLASTp (e-value cutoff 1E-10). This resulted in
169 B. dorsalis unique transcripts with high sequence similarity to at least one of the $D$. melanogaster peptide sequences (Additional file 5).

The genes Sex lethal (Sxl) and Notch $(N)$ had the highest number of transcripts with high sequence similarity in B. dorsalis. The gene transformer (tra) was not found in our transcriptome assembly, and only two transcripts matched transformer 2 (tra2) (which has 7 transcripts in D. melanogaster). The gene tra2 was found to be critical for sex-determiantion in Anastrepha fruit flies [27] but needs to interact with tra. While tra was found to be present in another Bactrocera species, $B$. oleae [28], it may not have been actively expressed in the samples we tested, despite our effort to include as many developmental stages as possible. A previous report investigated reproduction and development in $B$. dorsalis utilizing RNA-seq, but did not investigate sex-determination [11]. Additionally, putative sex determinant D. melanogaster orthologs and transcripts 


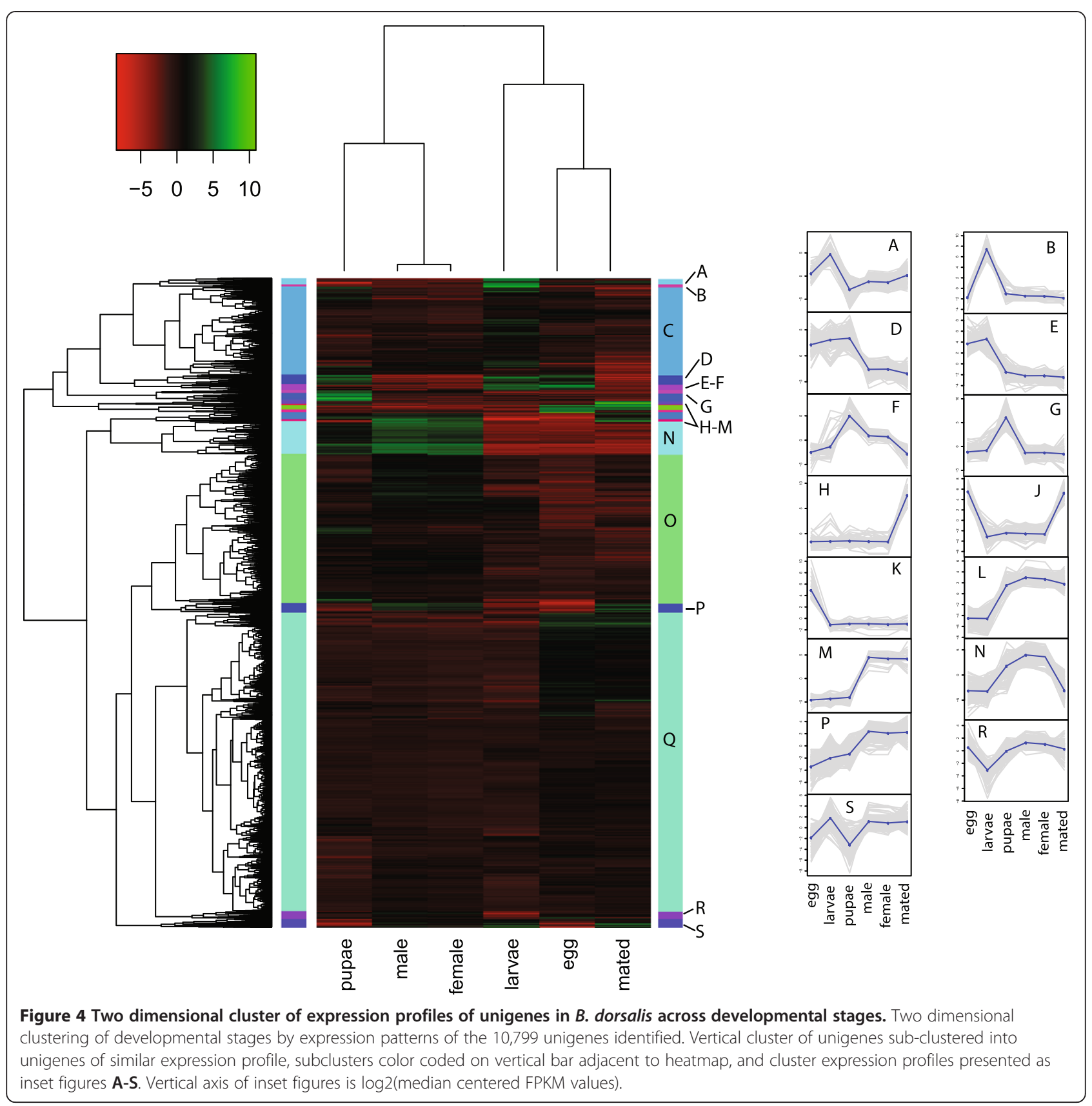

with sequence similarity to other species were mined from the annotation output and the $D$. melanogaster orthologous datasets. The total number of genes, transcripts and orthologs found are in Additional file 5.

\section{Conclusions}

The sequencing of stage-specific messenger RNA from the fruit fly $B$. dorsalis allowed for the construction of a transcriptome that when compared with $D$. melanogaster allowed for the identification of 20,666 transcripts across 10,799 unigenes. This is comparable to the current genome annotation derived D. melanogaster transcript set, which includes 15,504 genes, 25,205 isoforms, N50 isoform length of 3,633 and an isoform sum of $68.46 \mathrm{Mb}$. Utilizing binning expression nomenclature utilized by the modENCODE project, $65 \%$ of the unigenes identified were expressed at a constitutively low level (Figure 2). In addition, the relative distribution of unigenes across bins throughout the developmental life history in $B$. dorsalis is very similar in pattern to the distribution seen in D. melanogaster across the same developmental stages. Despite this, distribution does not directly demonstrate similarity between species at a gene or unigene level, so putative orthologs were identified using a reciprocal blast approach. 


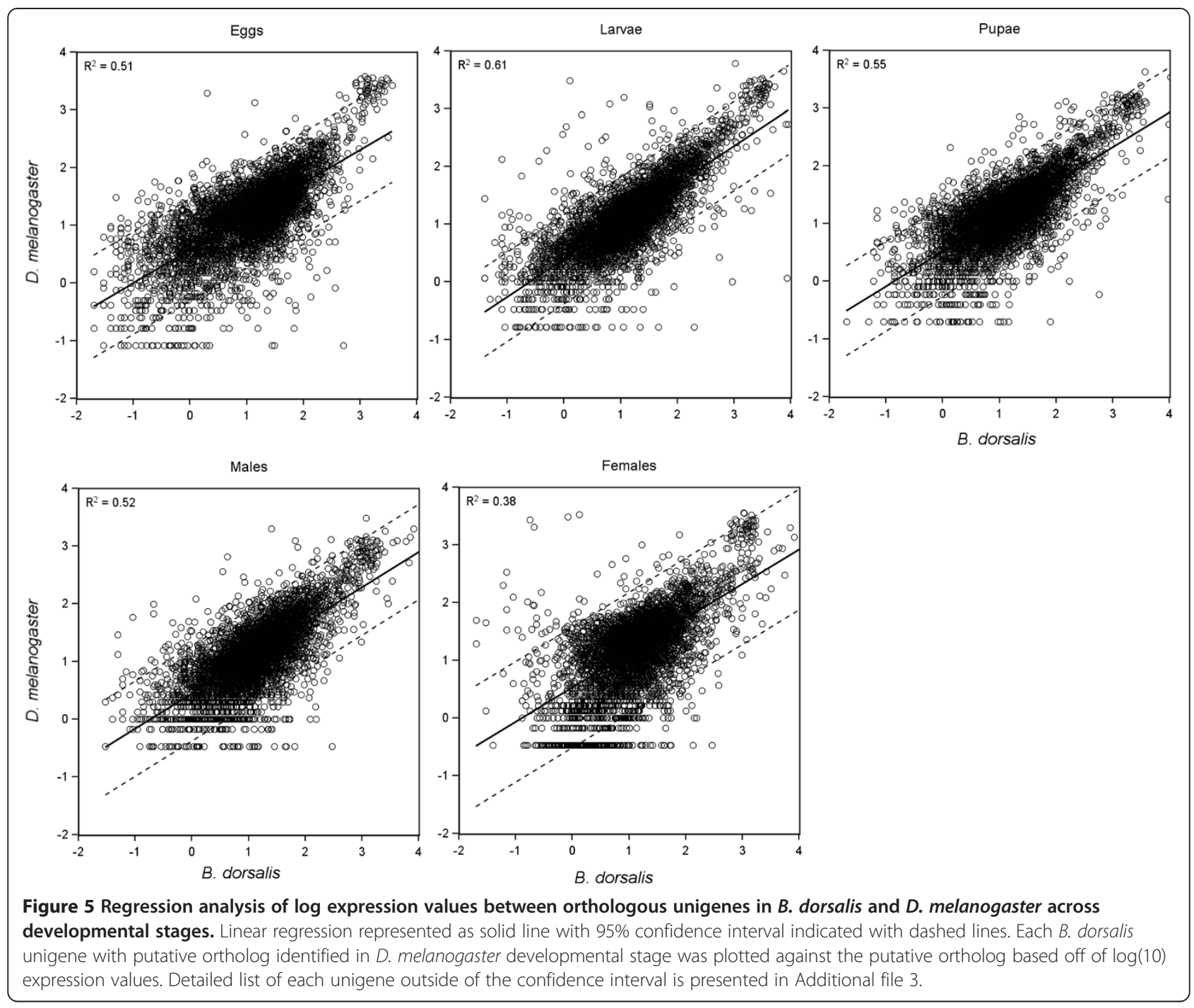

Functional annotation of the transcriptome yielded a large diversity of functions. The focus of this study was on proteins related to DNA binding, detoxification, and sex determination. This analysis demonstrates the utility of a D. melanogaster based annotation approach for closely related species, and potentially that functions or processes in Drosophila will largely translate to Tephritide flies. Despite this, direct transfer of Drosophila annotations is not always sufficient for inferring function. For example, genes involved in sex-determination are potential targets for the development of novel control strategies and the improvement of the sterile insect technique (SIT) in Tephritids. Orthology to Drosophila should be used with caution, as sex determination is highly diverged among dipeteran insects. For example, $S x l$ in tephritids is not regulated in a sex-specific fashion; rather the $S x l$ transcript is present in both sexes. To address this point, further studies utilizing more accurate and sensitive techniques will be needed to validate and verify the expression patterns of genes of interest throughout the life history of $B$. dorsalis. In addition, functional genomic approaches will lead to a better understanding of the similarities and differences between Tephritids and Drosophilids and serve as a foundation for developing new control techniques for pestiferous true fruit flies.

\section{Methods}

\section{Bactrocera dorsalis colony rearing and stage collections}

Bactrocera dorsalis eggs used in this experiment were obtained from the USDA-ARS-Pacific Basin Agricultural Research Center research colony "Punador" (Hilo, HI, USA) maintained on conventional mill feed diet [29]. This colony is derived from wild flies collected in Puna, Hawaii in 1984 and has since been maintained in the laboratory on artificial diet. These eggs were transferred to liquid based artificial diet $[30,31]$ and insects reared to obtain a collection of samples representing the entire life cycle of this insect. Typically, when reared on artificial diet in colony, eggs 
Table 3 Classification of transcripts annotated as transcription factors

\begin{tabular}{lcc}
\hline $\begin{array}{l}\text { Transcription factor } \\
\text { domain class }\end{array}$ & $\begin{array}{c}\text { Number of } \\
\text { transcripts }\end{array}$ & $\begin{array}{c}\text { Relative percentage of } \\
\text { total TF trascripts }\end{array}$ \\
\hline Zinc finger & 330 & 26.1 \\
Homeobox & 64 & 5.1 \\
Helix-loop-helix & 55 & 4.4 \\
BTB/POZ & 44 & 3.5 \\
Bzip & 39 & 3.1 \\
Myb & 24 & 1.9 \\
WD40 & 19 & 1.5 \\
Basal TFs & 18 & 1.4 \\
Fork-head & 18 & 1.4 \\
Bromodomain & 15 & 1.2 \\
Helicase & 13 & 1.0 \\
Other & 625 & 49.4 \\
Total & 1264 & 100.0 \\
\hline
\end{tabular}

Distribution of transcription factors. The eleven most abundant domain classes are listed, but 305 unique domains in total were identified. Transcripts belonging to classes not specifically listed were identified by fewer than 13 unique transcripts and pooled into the "other" category.

take approximately $1-3$ days to hatch; insects stay in the larval stage 8-10 days, are in pupal stage for 10 days, and survive as adults for several weeks. Maturation of adults takes approximately 7 days, and adults begin mating after that time. Samples were collected in the following manner in order to produce RNA samples that are representative of an entire life stage, rather than just a single point in time during development.

Fresh eggs from colony flies were maintained at room temperature for 3 days until complete hatch. During that time, a daily sample of eggs was taken. The samples were snap frozen in liquid nitrogen and then stored at $-80^{\circ} \mathrm{C}$. After hatch, 12 separate containers of liquid artificial diet were set up with larvae, maintained at room temperature, and each day for 10 days one container was used to collect larvae. Liquid diet containing larvae was strained through a mesh sieve and larvae were quickly rinsed in distilled water. Larvae were then collected in microfuge tubes and snap frozen in liquid nitrogen and stored at $-80^{\circ} \mathrm{C}$. The larvae in the remaining two containers were allowed to pupate following standard methods. Pupae were collected and daily pupal samples were collected for 10 days; each day pupae were snap frozen and stored. Adult cages with sugar water and torula yeast were then set up with mature pupae, and adults were allowed to emerge following standard protocols [32]. At this time, adults were sexed and males and females separated. Daily collections of virgin males and females were made for 7 days and samples immediately snap frozen. After that time, males and females were combined into a single cage
Table 4 Distribution of detoxification annotations in $B$. dorsalis transcriptome including cytochrome P450, glutathione S-transferases, and UDPglucuronosyltransferases

\begin{tabular}{|c|c|c|c|}
\hline & Gene class & Family & $\begin{array}{l}\text { Number found in } \\
\text { B. dorsalis }\end{array}$ \\
\hline \multirow[t]{22}{*}{ P450s } & P450 monooxygenases & CYPIV & 21 \\
\hline & & CYPIII & 1 \\
\hline & & CYPIX & 2 \\
\hline & & CYPVI & 29 \\
\hline & & CYPXII & 11 \\
\hline & & CYPXLIX & 1 \\
\hline & & CYPXVIII & 1 \\
\hline & & CYPXXVIII & 2 \\
\hline & & CYPCCCI & 1 \\
\hline & & CYPCCCIII & 1 \\
\hline & & CYPCCCIV & 2 \\
\hline & & CYPCCCIX & 4 \\
\hline & & CYPCCCV & 1 \\
\hline & & CYPCCCVI & 1 \\
\hline & & CYPCCCVIII & 1 \\
\hline & & CYPCCCX & 1 \\
\hline & & CYPCCCXI & 1 \\
\hline & & CYPCCCXIII & 6 \\
\hline & & CYPCCCXIV & 1 \\
\hline & & CYPCCCXV & 1 \\
\hline & & CYPCCCXVII & 1 \\
\hline & & CYPCCCXVIII & 2 \\
\hline GSTs & glutathione S-transferases & & $34^{\beta}$ \\
\hline UGTS & UDP-glucuronosyltransferases & & 23 \\
\hline
\end{tabular}

${ }^{a}$ Breakdown of individual unigenes annotated to each class available in Additional file 4.

${ }^{\beta}$ For GSTs, 4 contained only $\mathrm{N}$-terminal domains, 4 only C-terminal domains and 26 genes encompassed both domains.

for mating. Mating cages were carefully observed and mated females were identified, collected, and frozen.

\section{RNA extraction and sequencing}

Total RNA was extracted from each collection day spanning from egg to adult using the Qiagen RNeasy Plus Mini Kit (Qiagen Inc., Valencia, CA) following the manufacturer's procedures with the following modifications. Approximately $30-50 \mathrm{mg}$ of liquid nitrogen snap-frozen tissue was placed in $600 \mu \mathrm{l}$ Buffer RLT with $1 \% \beta$-mercaptoethanol and ground carefully with disposable micropestel in a microfuge tube. This solution was then passed through a QIAshredder column and then through a gDNA Eliminator column. In addition, before final elution, on column RNase-Free DNase treatment was performed to ensure full removal of genomic DNA 
from sample. RNA concentration and quality was assessed using a Qubit fluorometer (Invitrogen Corp., Carlsbad, CA, USA) as well as an Agilent 2100 Bioanalyzer (Santa Clara, CA, USA) following standard protocols. From these RNA extractions, stage-specific samples were created by pooling intra-stage samples at an equal concentration ratio. In total, six stage-representative samples were collected representing egg, larval, pupal, adult male, adult female, and mated adult female stages. Each of these total RNA samples was prepared for sequencing using the TruSeq RNA Sample Preparation Kit (Illumina Inc., San Diego, CA, USA) and sequenced on an Illumina GAIIx to produce approximately $14-20$ million $2 \times 101$ bp PE reads per sample library.

\section{Raw read quality filiter, in silico library normalization and de novo transcriptome assembly}

Pre-filtering the reads for quality is critical to obtaining a high quality assembly and produce accurate RNA-Seq expression data. Reads were filtered that contained a Phred score below 20 across more than $20 \%$ of the bases using the fastx-toolkit fastq_quality_filter script (http://hannonlab.cshl.edu/fastx_toolkit/commandline. html). These quality-filtered reads were then normalized to reduce redundant read data and discard read errors using Trinity's normalize_by_kmer_coverage.pl script with a kmer size of 25 and maximum read coverage of 30. The resulting normalized reads were used to create a de novo transcriptome assembly using the Trinity de novo transcriptome assembly pipeline (r2012-10-05) [19,33]. The Trinity pipeline (Inchworm, Chrysalis, and Butterfly) was executed using default parameters, implementing the -REDUCE flag in Butterfly and utilizing the Jellyfish k-mer counting approach [33]. Assembly completed in 3 hours and 13 minutes on a compute node consisting of 32 Xeon $3.1 \mathrm{GHz}$ cpus and $256 \mathrm{~Gb}$ of RAM available to the software on the USDA-ARS Pacific Basin Agricultural Research Center Moana computer cluster (http://moana. dnsalias.org).

\section{Assembly filtering and gene prediction}

The output of the Trinity pipeline is a fasta formatted file containing sequences defined as a set of transcripts, including alternatively spliced isoforms determined during graph reconstruction in the Butterfly step. These transcripts are grouped into gene components, which represent multiple isoforms across a single unigene model. While many full-length transcripts are expected to be present, it is likely that the assembly also consists of erroneous contigs, partial transcript fragments, and non-coding RNA molecules. This collection of sequences was thus filtered to identify contigs containing full or near full-length transcripts or likely coding regions and isoforms that are representative at a minimum level based off of read abundance. Pooled non-normalized reads were aligned to the unfiltered Trinity.fasta transcript file using bowtie 0.12.7 through the alignReads.pl script distributed with Trinity. Abundance of each transcript was calculated using RSEM 1.2.0 utilizing the Trinity wrapper run_RSEM.pl [13,34]. Through this wrapper, RSEM read abundance values were calculated on a per-isoform and per-gene basis. In addition, the percent composition of each transcript component for each gene is calculated. From these results, the original assembly file produced by Trinity was filtered to remove transcripts that represent less than $1 \%$ of the RSEM based expression level of its parent gene or transcripts with TPM (Transcripts per Million) value below 0.5 . This filter set discarded is referred to as "transcripts filtered: low abundance" in figures and tables and the retained transcripts used as input for further filtering.

Coding sequence was predicted from the filtered transcripts using the transcripts_to_best_scoring_ORFs.pl script distributed with the Trinity software from both strands of the transcripts. This approach uses the software Transdecoder (http://transdecoder.sourceforge.net/) which first identifies the longest open reading frame (ORF) for each transcript and then the 500 longest ORFs are used to build a Markov model against a randomization of these ORFs to distinguish between coding and non-coding regions. This model is then used to score the likelihood of the longest ORFs in all of the transcripts, reporting only those putative ORFs which outscore the other reading frames [13]. Thus, the low abundance filtered transcript assembly was split into contigs that contain complete transcripts (Retained: Full Length ORF), contigs containing transcript fragments with predicted partial ORF (Retained: Partial ORF) and contigs containing no ORF prediction (Transcripts filtered: non-coding). The resulting retained transcript sets (partial and full) were merged and subjected to annotation and utilized in subsequent analysis. In addition, sequence length and read count histograms were created for each of the four filter categories described above to help visualize the effect of filtering on retention of transcripts (Figure 1A-B).

\section{Gene annotation}

The filtered transcripts were annotated using the UniProtKB/Swiss-Prot database, Pfam-A, eggNOG, and gene ontology utilizing the Trinotate annotation pipeline. In addition, homologous proteins in Drosophila melanogaster were identified. The filtered transcript set was first subjected to blastp search against the UniProtKB/Swiss-Prot database using blast-2-2-26+ with an e-value cutoff of 1.0E-5. In addition, protein domains were identified through searching the Pfam_A database using HMMER 3.0. Signal peptides and transmembrane domains were annotated with SignalP 4.1 and TMHMM 2.0 respectively. The resulting outputs 
were loaded into a Trinotate database, where eggNOG and Gene Ontology terms were annotated and the resulting annotation set was exported as a delimited file for further analysis (Additional file 6). In addition, transcripts were subjected to BLASTx search against the current $D$. melanogaster protein set (Flybase.org, Dmel-r5.44) and uniref90 using an e-value cutoff of 1.0E-5 to identify homologous genes [35]. In addition, percent alignment length of the query transcript against the top $D$. melanogaster alignment was calculated and binned for each filtering category and plotted (Figure 1C). To further define putative orthologs to $D$. melanogaster proteins, reciprocal BLASTp alignment was performed between protein sequences in the $B$. dorsalis transcriptome assembly and all known $D$. melanogaster proteins. Putative orthologs were defined only when reciprocal top scoring hit (at unigene/gene level) between both searches were the same and were unique. This yielded 5,681 unigenes orthologous with $D$. melanogaster genes. An additional 4,186 unigenes were members of a reciprocal hit that was not unique, with multiple unigene/genes having equal score. These were not included as putative orthologs, as they may represent paralogous proteins or other situations without a 1:1 relationship, and thus this is a conservative, yet largely accurate analysis [36]. Putative orthologs are presented in Additional file 7, as a cross reference between flybase gene ID and $B$. dorsalis unigene identifier. The resulting annotated transcriptome was converted to Genbank .tbl format using a betarelease of the Genome Annotation Generator (http:// genomeannotation.github.io/GAG/) and transvestigator (http://genomeannotation.github.io/transvestigator/) and submitted to NCBI under Transcriptome Shotgun Assembly (TSA) GAKP00000000 associated with Bioproject 167923.

\section{Read library mapping and expression analysis}

Because the Trinity assembler is able to accurately predict splice isoforms, gene and isoform expression quantification was performed using the RSEM (RNA-Seq by Expectation Maximization) software package (v1.1.15) which is particularly well suited to work with multiple isoforms where the same read may map to multiple sequences [34]. The filtered transcript set from Trinity was used for analysis that only contained contigs containing likely coding sequence (full and partial ORFs) to avoid skewing expression quantification results with non-coding and fragmented data. Quality filtered reads from each sequencing library (egg, larvae, pupae, adult male, adult female, mated adult female) were independently mapped to the reference transcriptome assembly created by Trinity using bowtie (v 0.12.7) [37] using the alignReads.pl script distributed with Trinity. The resulting bam formatted mapping files were sorted and used to produce fragment abundance estimation by RSEM [34].
Transcript abundance values were produced as expected read count, and they were normalized using trimmed mean of $\mathrm{M}$ values (TMM) methods and transformed into fragments per feature kilobase per million reads mapped (FPKM) for each gene and the individual isoforms that compose each gene for each developmental library using scripts provided by Trinity [38,39]. These TMM normalized FPKM values were appended to the annotation information, and binned into expression level categories based off of FPKM values (No Expression, very low, low, moderate, moderately high, high, very high, and extremely high) following the approach and nomenclature used to describe modENCODE expression data in FlyBase2012_06 (David Emmert and William Gelbart, personal communication) (Figure 4, Additional file 1) $[14,35,40]$. TMM normalized FPKM values were also used to create a dissimilarity matrix based on Euclidean distance utilizing the dist function in $\mathrm{R}$ and this matrix was used to perform cluster analysis using the complete agglomeration method in the hclust $\mathrm{R}$ function. These clusters and the TMM normalized FPKM values were used to create a heatmap of expression levels sorted by both transcript and sample clusters utilizing the $\mathrm{R}$ enhanced heatmap (heatmap.2) function. Each cluster in the expression heatmap describes a different developmental expression pattern shared by all genes present in that cluster. Both TMM normalized and read count matrices are available under the NCBI Gene Expression Omnibus (GEO) under accession GSE46310 associated with BioProject PRJNA198716.

\section{Additional files}

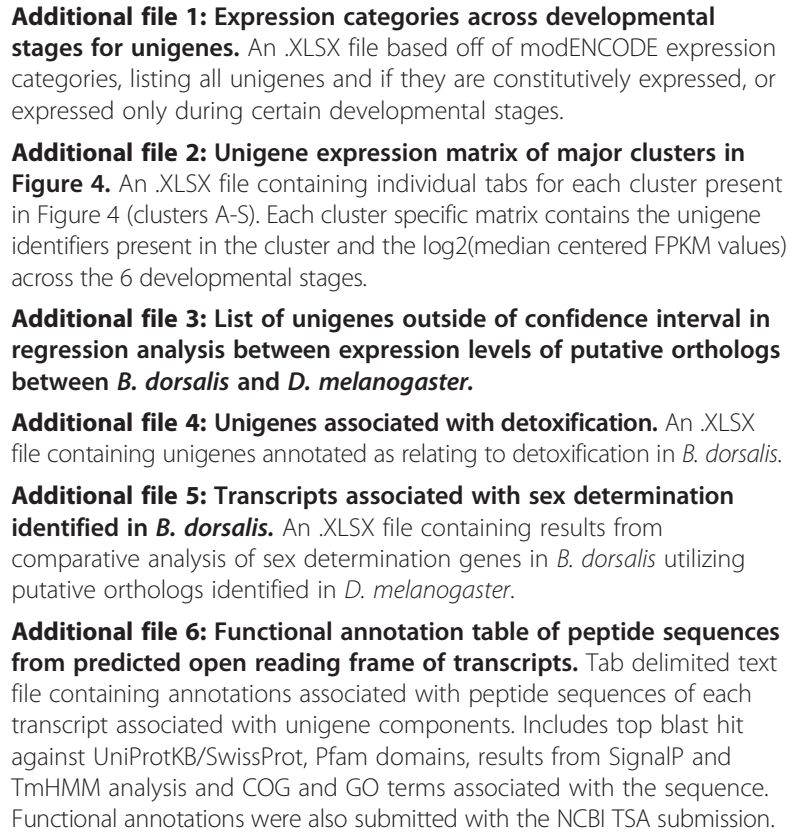

Additional file 2: Unigene expression matrix of major clusters in Figure 4. An .XLSX file containing individual tabs for each cluster present in Figure 4 (clusters A-S). Each cluster specific matrix contains the unigene identifiers present in the cluster and the log2(median centered FPKM values) across the 6 developmental stages.

Additional file 3: List of unigenes outside of confidence interval in regression analysis between expression levels of putative orthologs between $B$. dorsalis and $D$. melanogaster.

Additional file 4: Unigenes associated with detoxification. An .XLSX file containing unigenes annotated as relating to detoxification in $B$. dorsalis. Additional file 5: Transcripts associated with sex determination identified in $\boldsymbol{B}$. dorsalis. An .XLSX file containing results from comparative analysis of sex determination genes in $B$. dorsalis utilizing putative orthologs identified in D. melanogaster.

Additional file 6: Functional annotation table of peptide sequences from predicted open reading frame of transcripts. Tab delimited text file containing annotations associated with peptide sequences of each transcript associated with unigene components. Includes top blast hit against UniProtKB/SwissProt, Pfam domains, results from SignalP and TmHMM analysis and COG and GO terms associated with the sequence. Functional annotations were also submitted with the NCBI TSA submission. 
Additional file 7: Putative orthologous genes/unigenes between D. melanogaster and B. dorsalis. An .XLSX file containing B. dorsalis unigene identifier and D. melanogaster Flybase gene ID (FBgn\#\#\#\#\#\#). First tab presents putative orthologs as defined as reciprocal best unique blast hit, and second tab presents unigenes with top scoring hit, but not considered orthologous due to multiple top scoring unigenes/genes (non-unique hit)

\section{Competing interests}

The authors declare that they have no competing interests.

\section{Authors' contributions}

Conceived and designed the experiments: SMG. Performed the experiments: SMG, SH, BC. Analyzed the data: SMG, BC, BDH, SMG, and NM. Evaluated conclusions: SMG, BC, BDH, SH and NM. Contributed reagents/materials/ analysis tools: BC, BDH, SH, SMG, and NM. Wrote the paper: SMG and BC. All authors read and approved the final manuscript.

\section{Acknowledgements}

We thank Steven Tam for assistance in rearing and extraction of sample collections used in this study. Funding was provided by USDA-ARS and B.H. was supported by USDA Farm Bill Project 3.0251. Bioinformatic analysis was performed on computing resources at USDA-ARS Pacific Basin Agricultural Research Center (Moana cluster; Hilo, HI) and the Extreme Science and Engineering Discovery Environment (XSEDE), which is supported by National Science Foundation grant number OCl-1053575XSEDE utilizing allocation TG-MCB140032 to SMG. Opinions, findings, conclusions, or recommendations expressed in this publication are those of the authors and do not necessarily reflect the views of the USDA. USDA is an equal opportunity provider and employer

\section{Author details}

${ }^{1}$ Tropical Crop and Commodity Protection Research Unit, USDA-ARS Daniel K Inouye Pacific Basin Agricultural Research Center, 64 Nowelo Street, Hilo, HI 96720, USA. ²Department of Plant and Environmental Protection Sciences, University of Hawaii, Manoa, Honolulu, HI, USA. ${ }^{3}$ Advanced Studies in Genomics, Proteomics and Bioinformatics, University of Hawai'i Manoa, Honolulu, HI 96822, USA

Received: 28 May 2014 Accepted: 2 October 2014

Published: 28 October 2014

\section{References}

1. Allwood AJ, Chinajariyawong A, Kritsaneepaiboon S, Drew RAl, Hamacek EL, Hancock DL, Hengsawad C, Jipanin JC, Jirasurat M, Krong CK, Leong CTS, Vijaysegaran S: Host plant records for fruit flies (Diptera: Tephritidae) in Southeast Asia. Raffles Bull Zool 1999, 47:1-92.

2. Hardy DE: Taxonomy and distribution of the oriental fruit fly and related species (Tephritidae, Diptera). Proc Hawaiian Entomol Soc 1969, 20:395-428.

3. Hardy DE: Studies in Hawaiian fruit flies (Diptera, Tephritidae). Proc Entomol Soc Wash 1949, 51:181-205.

4. Armitage HM: Susceptibility of California fruits and vegetables to attack by the Oriental fruit fly (Dacus dorsalis). Bull Dep Agric Calif 1951, 40:1-9.

5. Fullaway DT: The Oriental fruit fly (Dacus dorsalis Hendel) in Hawaii. Proc Pac Sci Congr VII 1953, 4:148-163.

6. Wiegmann BM, Trautwein MD, Winkler IS, Barr NB, Kim J-W, Lambkin C, Bertone MA, Cassel BK, Bayless KM, Heimberg AM, Wheeler BM, Peterson K, Pape T, Sinclair BJ, Skevington JH, Blagoderov V, Caravas J, Kutty SN, Schmidt-Ott U, Kampmeier GE, Thompson FC, Grimaldi DA, Beckenbach AT, Courtney GW, Friedrich M, Meier R, Yeates DK: Episodic radiations in the fly tree of life. Proc Natl Acad Sci 2011, 108(17):5690-5695.

7. Yang $Y$, Smith S: Optimizing de novo assembly of short-read RNA-seq data for phylogenomics. BMC Genomics 2013, 14(1):328.

8. O'Neil S, Emrich S: Assessing De Novo transcriptome assembly metrics for consistency and utility. BMC Genomics 2013, 14(1):465.

9. Grabherr MG, Haas BJ, Yassour M, Levin JZ, Thompson DA, Amit I, Adiconis X, Fan L, Raychowdhury R, Zeng Q, Chen Z, Mauceli E, Hacohen N, Gnirke A Rhind N, di Palma F, Birren BW, Nusbaum C, Lindblad-Toh K, Friedman N,
Regev A: Full-length transcriptome assembly from RNA-Seq data without a reference genome. Nat Biotechnol 2011, 29(7):644-652

10. Shen G-M, Dou W, Niu J-Z, Jiang H-B, Yang W-J, Jia F-X, Hu F, Cong L, Wang J-J: Transcriptome analysis of the oriental fruit fly (Bactrocera dorsalis). Plos One 2011, 6(12):e29127.

11. Zheng W, Peng T, He W, Zhang H: High-throughput sequencing to revea genes involved in reproduction and development in Bactrocera dorsalis (Diptera: Tephritidae). PLoS ONE 2012, 7(5):e36463.

12. Hsu J-C, Chien T-Y, Hu C-C, Chen M-JM, Wu W-J, Feng H-T, Haymer DS, Chen C-Y: Discovery of genes related to insecticide resistance in Bactrocera dorsalis by functional Genomic analysis of a De novo assembled transcriptome. Plos One 2012, 7(8):e40950.

13. Haas BJ, Papanicolaou A, Yassour M, Grabherr M, Blood PD, Bowden J, Couger MB, Eccles D, Li B, Lieber M, MacManes MD, Ott M, Orvis J, Pochet N, Strozzi F, Weeks N, Westerman R, William T, Dewey CN, Henschel R, LeDuc RD, Friedman N, Regev A: De novo transcript sequence reconstruction from RNA-seq using the Trinity platform for reference generation and analysis. Nat Protoc 2013, 8(8):1494-1512.

14. Roy S, Ernst J, Kharchenko PV, Kheradpour P, Negre N, Eaton ML, Landolin JM, Bristow CA, Ma L, Lin MF, Washietl S, Arshinoff Bl, Ay F, Meyer PE, Robine N, Washington NL, Di Stefano L, Berezikov E, Brown CD, Candeias R, Carlson JW, Carr A, Jungreis I, Marbach D, Sealfon R, Tolstorukov MY, Will S, Alekseyenko AA, Artieri C, Booth BW, et al: Identification of functional elements and regulatory circuits by Drosophila modENCODE. Science 2010, 330(6012):1787-1797.

15. FlyBase: FlyBase: a drosophila database. Nucleic Acids Res 1998 26(1):85-88.

16. Adryan B, Teichmann SA: FlyTF: a systematic review of site-specific transcription factors in the fruit fly Drosophila melanogaster. Bioinformatics 2006, 22(12):1532-1533.

17. Feyereisen R: Insect P450 enzymes. Annu Rev Entomol 1999, 44:507-533.

18. Guengerich FP: Cytochrome p450 and chemical toxicology. Chem Res Toxicol 2008, 21(1):70-83

19. Shen GM, Dou W, Huang $Y$, Jiang XZ, Smagghe G, Wang JJ: In silico cloning and annotation of genes involved in the digestion, detoxification and RNA interference mechanism in the midgut of Bactrocera dorsalis Hendel (Diptera: Tephritidae). Insect Mol Biol 2013, 22(4):354-365

20. Huang $Y$, Shen G-M, Jiang H-B, Jiang X-Z, Dou W, Wang J-J: Multiple P450 genes: Identification, tissue-specific expression and their responses to insecticide treatments in the oriental fruit fly, Bactrocera dorsalis (Hendel) (Diptera: Tephritidea). Pestic Biochem Physiol 2013, 106(1-2):1-7.

21. Pavlidi N, Dermauw W, Rombauts S, Chrisargiris A, Van Leeuwen T, Vontas J: Analysis of the Olive Fruit Fly Bactrocera oleae Transcriptome and Phylogenetic Classification of the Major Detoxification Gene Families. Plos One 2013, 8(6):e66533.

22. Misra JR, Horner MA, Lam G, Thummel CS: Transcriptional regulation of xenobiotic detoxification in Drosophila. Genes Dev 2011, 25(17):1796-1806.

23. Mishra M, Sharma A, Negi MP, Dwivedi UN, Chowdhuri DK: Tracing the tracks of genotoxicity by trivalent and hexavalent chromium in Drosophila melanogaster. Mutat Res 2011, 722(1):44-51.

24. Franz G, Robinson AS: Molecular technologies to improve the effectiveness of the sterile insect technique. Genetica 2011, 139(1):1-5.

25. Ding $Y$, Zhao L, Yang S, Jiang Y, Chen Y, Zhao R, Zhang Y, Zhang G, Dong Y, Yu H, Zhou Q, Wang W: A young Drosophila duplicate gene plays essential roles in spermatogenesis by regulating several Y-linked male fertility genes. PLoS Genet 2010, 6(12):e1001255.

26. Salz H, Erickson JW: Sex determination in Drosophila: The view from the top. Fly 2010, 4(1):60-70.

27. Sarno F, Ruiz M, Eirin-Lopez J, Perondini A, Selivon D, Sanchez L: The gene transformer-2 of Anastrepha fruit flies (Diptera, Tephritidae) and its evolution in insects. BMC Evol Biol 2010, 10(1):140.

28. Lagos D, Koukidou M, Savakis C, Komitopoulou K: The transformer gene in Bactrocera oleae: the genetic switch that determines its sex fate. Insect Mol Biol 2007, 16(2):221-230.

29. Tanaka N, Steiner LF, Ohinata K, Okamoto R: Low-cost larval rearing medium for mass production of oriental and Mediterranean fruit flies. J Econ Entomol 1969, 62(4):967-968.

30. Chang $C L$, Caceres $C$, Jang EB: A novel liquid larval diet and its rearing system for melon fly, Bactrocera cucurbitae (Diptera: Tephritidae). Ann Entomol Soc Am 2004, 97(3):524-528. 
31. Chang CL, Vargas Rl, Caceres C, Jang E, Cho IK: Development and assessment of a liquid larval diet for Bactrocera dorsalis (Diptera: Tephritidae). Ann Entomol Soc Am 2006, 99(6):1191-1198.

32. Spencer JP, Fujita BH: Oriental fruit fly, Bactrocera dorsalis (Hendel), A procedural manual for mass rearing four species of tephritid fruit flies. USDA, Agricultural Research Service: Honolulu, HI; 1997.

33. Marçais G, Kingsford C: A fast, lock-free approach for efficient parallel counting of occurrences of k-mers. Bioinformatics 2011, 27(6):764-770.

34. Li B, Dewey C: RSEM: accurate transcript quantification from RNA-Seq data with or without a reference genome. BMC Bioinformatics 2011, 12(1):323.

35. Drysdale RA, Crosby MA, FlyBase C: FlyBase: genes and gene models. Nucleic Acids Res 2005, 33:D390-D395.

36. Moreno-Hagelsieb G, Latimer K: Choosing BLAST options for better detection of orthologs as reciprocal best hits. Bioinformatics 2008, 24(3):319-324.

37. Langmead B, Trapnell C, Pop M, Salzberg SL: Ultrafast and memory-efficient alignment of short DNA sequences to the human genome. Genome Biol 2009, 10(3):R35.

38. Robinson $M$, Oshlack A: A scaling normalization method for differential expression analysis of RNA-seq data. Genome Biol 2010, 11(3):R25.

39. Mortazavi A, Williams BA, McCue K, Schaeffer L, Wold B: Mapping and quantifying mammalian transcriptomes by RNA-Seq. Nat Meth 2008 5(7):621-628.

40. Graveley BR, Brooks AN, Carlson JW, Duff MO, Landolin JM, Yang L, Artieri CG, van Baren MJ, Boley N, Booth BW, Brown JB, Cherbas L, Davis CA, Dobin A, Li R, Lin W, Malone JH, Mattiuzzo NR, Miller D, Sturgill D, Tuch BB, Zaleski C, Zhang D, Blanchette M, Dudoit S, Eads B, Green RE, Hammonds A, Jiang L, Kapranov $P$, et al: The developmental transcriptome of Drosophila melanogaster. Nature 2011, 471(7339):473-479.

doi:10.1186/1471-2164-15-942

Cite this article as: Geib et al:: Characterizing the developmental transcriptome of the oriental fruit fly, Bactrocera dorsalis (Diptera: Tephritidae) through comparative genomic analysis with Drosophila melanogaster utilizing modENCODE datasets. BMC Genomics 2014 15:942.

\section{Submit your next manuscript to BioMed Central and take full advantage of:}

- Convenient online submission

- Thorough peer review

- No space constraints or color figure charges

- Immediate publication on acceptance

- Inclusion in PubMed, CAS, Scopus and Google Scholar

- Research which is freely available for redistribution 